\section{INFLUENCIA DEL GÉNERO, NIVEL EDUCATIVO Y PRÁCTICA DEPORTIVA DE LOS PROGENITORES SOBRE HÁBITOS DEPORTIVOS EN ESCOLARES}

INFLUÊNCIA DO GÊNERO, NÍVEL EDUCACIONAL E PRÁTICA DESPORTIVA DOS PAIS NOS HÁBITOS ESPORTIVOS DAS CRIANÇAS EM IDADE ESCOLAR C

INFLUENCE OF GENDER, EDUCATIONAL LEVEL AND PARENTS' SPORTS PRACTICE ON SPORTS HABITS OF SCHOOL CHILDREN C?

doi' https://doi.org/10.22456/1982-8918.109610

(iD) Adrián Mateo-Orcajada* <amateo5@alu.ucam.edu>

(iD) Raquel Vaquero-Cristóbal* <rvaquero@ucam.edu>

Lucía Abenza-Cano* <labenza@ucam.edu>

Sonia María Martínez-Castro*<smmartinez@ucam.edu>

Ana María Gallardo-Guerrero* <amgallardo@ucam.edu>

(iD) Alejandro Leiva-Arcas* <aleiva@ucam.edu>

(D) Antonio Sánchez-Pato* <apato@ucam.edu>

*Universidad Católica San Antonio de Murcia, Murcia, España.

Resumen: La importancia de los padres en la actividad física de sus hijos durante la adolescencia ha sido constatada previamente, pero pocos estudios han establecido cuáles son las variables más determinantes. El objetivo del presente estudio fue analizar las diferencias en la práctica deportiva de los adolescentes según el nivel educativo, el nivel de práctica deportiva y los estereotipos de género de sus progenitores. La muestra estuvo compuesta por 965 adolescentes y 1599 progenitores de la Región de Murcia. Los resultados mostraron diferencias en la práctica deportiva de los adolescentes al considerar el nivel educativo de las madres, la actividad física realizada por los padres para los varones, la frecuencia de práctica de las madres para las mujeres y los estereotipos de género de las madres. Como conclusiones, señalar que los estereotipos de género y el nivel educativo de los progenitores influyeron en la práctica deportiva de los adolescentes.

Palabras clave: Ejercicio físico. Adolescente. Estereotipo de género. Padres.
Recibido en: 04 dec. 2020 Aprobado en: 17 ago. 2021 Publicado en: 08 oct. 2021

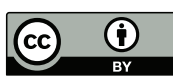

Este es un artículo publicado bajo la licencia Creative Commons Atribución 4.0 Internacional (CC BY 4.0).

eISSN: $1982-8918$ 


\section{INTRODUCCIÓN}

La inactividad física se ha convertido en uno de los problemas de salud pública más importantes a nivel mundial, siendo el cuarto factor de riesgo para la mortalidad (ORGANIZACIÓN MUNDIAL DE LASALUD, 2010). Esto es especialmente importante en la etapa de crecimiento ya que la inactividad física en la infancia y adolescencia predice, en gran medida, los comportamientos sedentarios en la etapa adulta (GONZALO-ALMOROX; URBANOS-GARRIDO, 2016; MAGALHAES; PEREIRA DE PINA; PEREIRA RAMOS, 2017). Así, se ha encontrado que en los adolescentes el sedentarismo se asocia con un mayor riesgo de sufrir enfermedades crónicas en la etapa adulta (RENNINGER et al., 2020).

Los agentes sociales con los que los adolescentes llevan a cabo actividades deportivas, en especial amigos y compañeros, cobran gran importancia para aumentar los niveles de práctica de los mismos (LISINSKIENE; JUSKELIENE, 2019). No obstante, los adolescentes también pueden verse influenciados por otros agentes sociales como los centros escolares (TAYLOR et al., 2010), los entrenadores (GUAGLIANO et al., 2014), los medios de comunicación (FERNÁNDEZ-PRIETO; GINÉ-GARRIGA; VÉLEZ, 2019) o los progenitores (SÁNCHEZ-ZAMORANO et al., 2019). En este sentido, los progenitores son determinantes, ya que pueden modificar los niveles de actividad física de los adolescentes (MENDONÇA et al., 2014), a pesar de que su influencia disminuye al incrementar la edad de sus hijos (GATOUILLAT; GRIFFET; TRAVERT, 2019).

Se ha reportado la importancia de numerosos factores en esta relación siendo el nivel educativo, la práctica deportiva realizada y los estereotipos de género de los progenitores factores destacados por su influencia sobrela práctica delos adolescentes. Respecto a la influencia del nivel educativo, se han encontrado resultados opuestos ya que mientras que Sanz-Arazuri et al. (2018) no hallaron diferencias en la práctica realizada por los adolescentes al considerar el nivel educativo de sus progenitores, Bringolf-Isler et al. (2018) concluyeron que un nivel educativo más alto de los padres, pero no de las madres, se relacionaba con niveles de actividad física más elevados en adolescentes; mientras que Magalhaes et al. (2017) y Steenholt et al. (2018), añadieron que tanto padres como madres con niveles educativos superiores, influían en la actividad física de los adolescentes en mayor medida que los que tenían niveles educativos más bajos. Estas diferencias halladas en la literatura científica dificultan extraer una conclusión sobre la influencia del nivel educativo de los progenitores en la práctica deportiva de los adolescentes.

En cuanto a la práctica de actividad física de los progenitores, numerosos estudios han tratado de relacionar la actividad física realizada por padres y madres con la realizada por los adolescentes. Los resultados obtenidos en las investigaciones previas son diversos ya que Bringolf-Isler et al. (2018) y Lisinskiene y Juskeliene (2019) hallaron que la actividad física de ambos progenitores influía significativamente en la práctica de los adolescentes, mientras que Cheng, Mendonça y Farias Júnior (2014) determinaron que había diferencias en función del sexo de los progenitores, siendo los padres más determinantes en la actividad física de los chicos y las madres en la de las chicas. 
Además, la frecuencia y duración de la práctica también se ven incrementadas cuando los adolescentes perciben que sus padres destinan más tiempo y días a la práctica de actividades físicas (SÁNCHEZ-ZAMORANO et al., 2019; RODRIGUES; PADEZ; MACHADO-RODRIGUES, 2018). En este sentido, la frecuencia y duración de práctica de los progenitores parece clave en la práctica de sus hijos, pero no hay información suficiente al respecto ya que Cheng, Mendonça y Farias-Júnior (2014) y Rodrigues, Padez y Machado-Rodrigues (2018) hallaron que los padres tenían mayor influencia en la frecuencia y duración de sus hijos, mientras que las madres la tenían en la de sus hijas; Lisinskiene y Juskeliene (2019) determinaron que los chicos mostraban influencia de los progenitores, pero no las chicas; y Wilk et al. (2018) afirmaron que la práctica de los adolescentes no presentaba diferencias significativas al considerar la de los progenitores. Además, el nivel de práctica de las chicas parece estar influido en mayor medida que el de los chicos (HENRIKSEN et al., 2016). Por tanto, la frecuencia y duración de práctica de los progenitores parece estar relacionada con la de los adolescentes, pero la información disponible en la literatura científica no permite extraer conclusiones concisas ya que cada uno de los progenitores podría influir en aspectos diferentes de la misma (CHENG; MENDONÇA; FARIAS-JUNIOR, 2014; LISINSKIENE; JUSKELIENE, 2019).

La influencia de los estereotipos de género de los progenitores también ha sido constatada en investigaciones previas en las que se halló que los adolescentes estaban influidos por los estereotipos de sus progenitores, lo que llevaba a muchos de ellos a practicar determinadas actividades deportivas y abandonar otras (BOICHÉ et al., 2014). Las chicas son el colectivo más afectado, ya que intentan practicar deportes socialmente considerados "femeninos", y en caso de no hacerlo, son cuestionadas por la sociedad, lo que conduce al abandono deportivo (CRANE; TEMPLE, 2014; LÓPEZALBALÁ, 2016), aunque el número de chicas que practican deportes "masculinos" en los últimos años está incrementando (ALVARIÑAS-VILLAVERDE et al., 2017; TOFFOLETTI; THORPE, 2018). No obstante, las chicas no son el único colectivo afectado, ya que los chicos también se ven influenciados por esta presión cuando realizan deportes considerados por la sociedad "poco masculinos" (ALVARIÑASVILLAVERDE et al., 2017; CHALABAEV et al., 2013).

Pero, los padres y madres parecen no ser conscientes de la influencia que pueden ejercer sobre las decisiones de sus hijos (BOICHÉ et al., 2014). Así, los progenitores indican que la falta de tiempo y la economía familiar son los motivos más determinantes para que sus hijos no practiquen actividad física (RODRIGUES; PADEZ; MACHADO-RODRIGUES, 2019), pero no tienen en cuenta la influencia que sus acciones, pensamientos y actitudes pueden tener sobre la práctica de los adolescentes, por lo que se requieren nuevas investigaciones que permitan ampliar la información al respecto (BOICHÉ et al., 2014). Por tanto, el objetivo del presente estudio fue analizar las diferencias en el nivel, frecuencia y duración de la práctica deportiva de los adolescentes según el nivel educativo, el nivel de práctica deportiva y los estereotipos de género de sus progenitores. 


\section{METODOLOGÍA}

\subsection{DISEÑO}

El diseño del presente estudio fue descriptivo y transversal, y constituye un primer ensayo para analizar la influencia del género, el nivel educativo y la práctica deportiva de los progenitores sobre hábitos deportivos en escolares desde un punto de vista multifactorial y en una gran muestra. Tanto para el diseño como para el desarrollo del manuscrito se siguió la declaración de STROBE (VANDENBROUCKE et al., 2014).

Previo a la investigación se obtuvo la aprobación del comité de ética institucional [código CE071924]. Además, todos los progenitores participantes en la investigación firmaron el consentimiento informado de forma previa a la toma de datos, donde se les informó de los objetivos del estudio, así como del tratamiento de los datos obtenidos y la confidencialidad de los mismos.

\subsection{PARTICIPANTES}

El tamaño mínimo de la muestra atendiendo a la ecuación para poblaciones finitas, con un tamaño de muestra válido para un intervalo de confianza del $95 \%$ fue de 382 estudiantes (MARTÍNEZ-ALMECIJA; MUÑOZ-GARCÍA; PASCUAL-ACOSTA, 2004). Considerando que la población de ESO de la Región de Murcia era de 69.888 estudiantes, se contó con una muestra inicial representativa compuesta de 2.715 estudiantes, con edades comprendidas entre 12 y 16 años, pertenecientes a nueve institutos de ESO de la Región de Murcia elegidos por conveniencia para obtener datos de todas las áreas de la Comunidad Autónoma. La selección de participantes dentro de estos centros se llevó a cabo mediante muestreo no probabilístico consecutivo, seleccionando a todos los sujetos posibles a los que se tuvo acceso, que querían participar voluntariamente en este estudio y que cumplieron los siguientes criterios de inclusión: 1) pertenecer a la ESO; 2) incluir el cuestionario del alumno junto al menos uno de los cuestionarios de los padres cumplimentados en su totalidad; y 3) aportar el consentimiento informado. La participación final fue de 965 alumnos, siendo 398 chicos y 567 chicas (media de edad: $13.92 \pm 1.39$ años) y de 1.599 progenitores, 716 padres (media de edad: $46.53 \pm 6.57$ años) y 883 madres (media de edad: $44.07 \pm 6.31$ años) (Figura 1). 
Figura 1 - Diagrama de flujo de la muestra

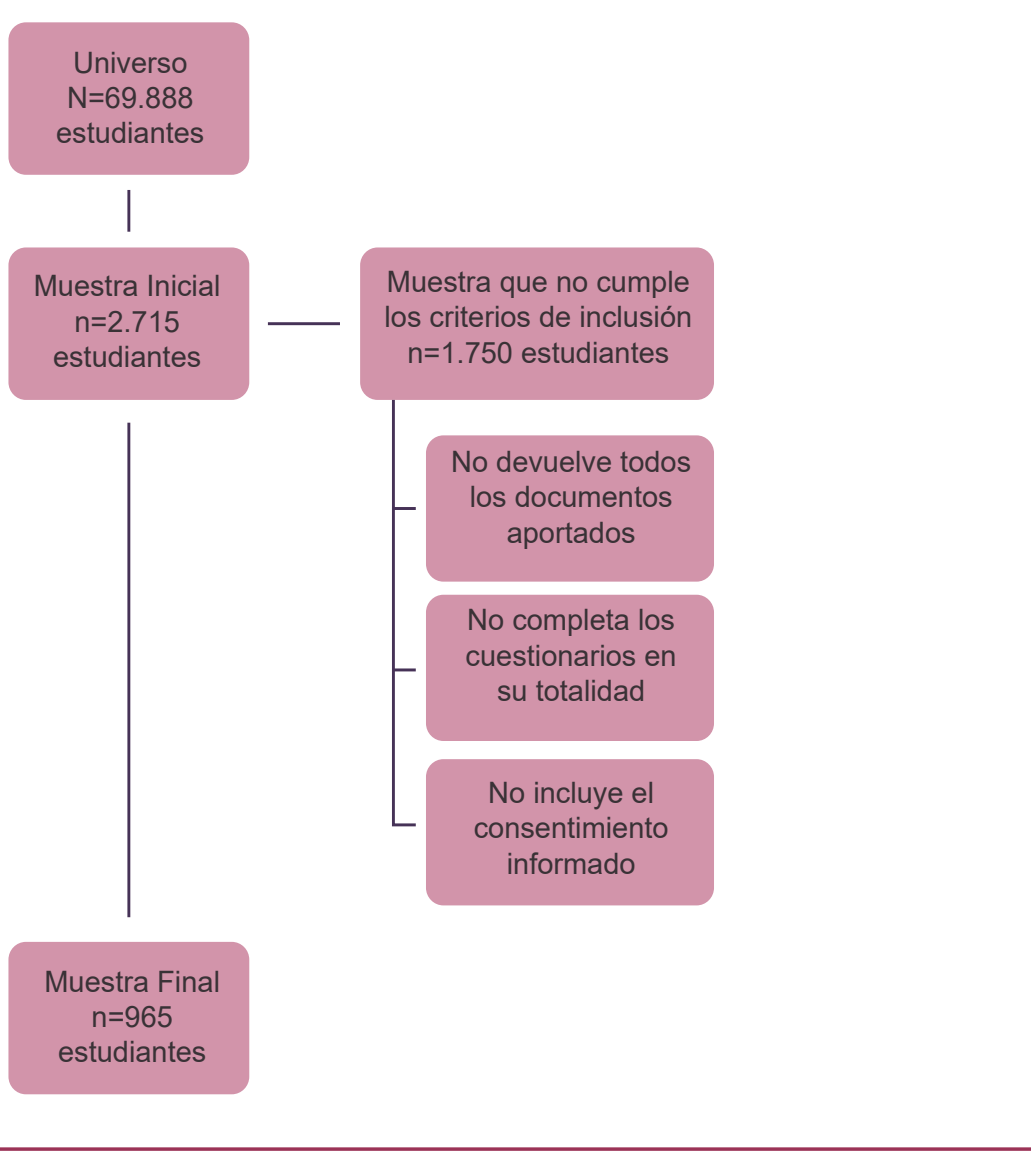

Fuente: Elaboración propia

\subsection{PROCEDIMIENTO}

En primer lugar, los investigadores del presente estudio realizaron una selección de nueve centros de ESO de la Región de Murcia que permitiera una muestra representativa de toda la Región y proporcional a la distribución de la población en edad escolar. Tras hablar con los equipos directivos, quienes dieron su conformidad a la realización del presente proyecto, se procedió a la toma de datos. Para ello el investigador principal entregó al responsable del área de Educación Física una copia de una carta de presentación informativa del proyecto; una carta con las instrucciones para la cumplimentación de los mismos por parte de alumnos y progenitores; una copia del consentimiento informado; una copia del cuestionario "Estilo de vida deportiva con perspectiva de género" (ALVARIÑAS-VILLAVERDE; FERNÁNDEZ-VILLARINO; LÓPEZ-VILLAR, 2009) a autocumplimentar por el alumno; y dos unidades del cuestionario "Creencias y estereotipos de género hacia la actividad física y deporte (CEGAFD)" (VERA; ARREBOLA; GARCÍA, 2018), a autocumplimentar por los progenitores del alumno. Tanto los adolescentes como sus progenitores tuvieron que responder a los cuestionarios de manera individual y anónima. Además, se insistió tanto a progenitores como a discentes sobre la importancia de la veracidad de las respuestas. La participación de los alumnos y sus progenitores fue totalmente voluntaria. 


\subsection{INSTRUMENTOS}

Para llevar a cabo la toma de datos, todos los alumnos cumplimentaron el cuestionario "Estilo de vida deportiva con perspectiva de género" (ALVARIÑASVILLAVERDE; FERNÁNDEZ-VILLARINO; LÓPEZ-VILLAR, 2009). Este instrumento aborda el pensamiento y las actitudes de los estudiantes acerca de la actividad física y el deporte (actividad física practicada, modalidad de actividad física, práctica de deporte escolar, actividades que les gustaría practicar, motivos de abandono de la actividad física, diferencias de género en la práctica, etc.). Se trata de un cuestionario semiestructurado compuesto por veinte preguntas, siendo la mayor parte de ellas cerradas. El valor correspondiente a los estereotipos de género de los adolescentes se obtuvo a través de doce ítems que se valoraron mediante una escala dicotómica de "acuerdo" o "desacuerdo". Para determinar el valor final se sumó la puntuación de los doce ítems pertenecientes a esta pregunta. En los ítems 5, 7 y 12, la respuesta "de acuerdo" recibió una puntuación de 1 , mientras que la de "desacuerdo" recibió una puntuación de 2; mientras que en los ítems 1, 2, 3, 4, 6, 8, 9, 10 y 11 la respuesta "de acuerdo" recibió una puntuación de 2 , mientras que la de "desacuerdo" recibió una puntuación de 1. La puntuación máxima de este apartado fue de 24 (ALVARIÑASVILLAVERDE; FERNÁNDEZ-VILLARINO; LÓPEZ-VILLAR, 2009).

Los progenitores cumplimentaron el cuestionario "Creencias y estereotipos de género hacia la actividad física y deporte (CEGAFD)" (VERA; ARREBOLA; GARCÍA, 2018), a través del que se obtuvo información sobre los hábitos de práctica deportiva (frecuencia, duración, tipo de práctica), nivel educativo y estereotipos de género de los mismos. El mismo cuenta con un total de 24 ítems que permiten evaluar cinco factores relacionados con los estereotipos de género: diferencias asociadas al género y su relación con la actividad física y el deporte; deporte y género; estereotipos sobre la actividad física y deporte asociados al género; creencias sobre la actividad física, deporte y género; $y$, las clases de Educación Física y el género. Cada ítem se contesta mediante una escala Likert de cuatro puntos según la intensidad ( 1 = totalmente en desacuerdo; 2 = en desacuerdo; 3 = de acuerdo; 4 = totalmente de acuerdo). Para determinar el valor correspondiente a cada uno de los cinco factores se sumó el valor aportado, de 1 a 4, a cada pregunta de esa dimensión siguiendo la metodología de Vera et al. (2018). La puntuación máxima de este test fue 96. La puntuación máxima en la categoría "diferencias asociadas al género y su relación con la actividad física y el deporte" fue 28, en "estereotipos sobre la actividad física y deporte asociados al género" 20 y en "deporte y género", "creencias sobre la actividad física, deporte y género" y "las clases de educación física y el género" 16 (VERA; ARREBOLA; GARCÍA, 2018).

\subsection{ANÁLISIS DE DATOS}

La distribución de los datos fue valorada inicialmente mediante el test de normalidad de Kolmogórov-Smirnov. Puesto que las variables seguían una distribución normal se realizó un análisis estadístico en base a prueba paramétricas. Para la obtención de los resultados se realizó una estadística descriptiva con la que se hallaron los valores medios de la muestra. Se realizó un análisis $X^{2}$ para establecer 
la influencia del nivel educativo de los progenitores en el nivel y tipo de práctica de los adolescentes; para hallar la influencia del nivel de práctica, la frecuencia y duración de los progenitores sobre el nivel de práctica, frecuencia y duración de los adolescentes; y para determinar la influencia de los estereotipos de género de los progenitores sobre el nivel de práctica deportiva de los adolescentes. Se utilizó el coeficiente de correlación de Pearson para analizar la relación existente entre los estereotipos de los progenitores y los adolescentes. Se estableció un valor de $p<0.05$ para determinar la significación estadística. El análisis estadístico fue realizado mediante el paquete estadístico SPSS (v. 25.0; SPSS Inc., IL).

\section{RESULTADOS}

El objetivo de la presente investigación fue analizar las diferencias en el nivel, frecuencia y duración de la práctica deportiva de los adolescentes según el nivel educativo, el nivel de práctica deportiva y los estereotipos de género de sus progenitores. Los resultados obtenidos presentan diferencias en el nivel de actividad realizada por los adolescentes en función del nivel educativo de sus progenitores (Tabla 1). Cabe destacar que el nivel de actividad física realizada por los adolescentes mostró diferencias significativas al considerar el nivel educativo de las madres, pero no el de los padres. En este sentido, un nivel educativo superior de las madres se relaciona positivamente con mayor práctica de los adolescentes.

Tabla 1 - Actividad física realizada por los adolescentes en función del nivel educativo de los padres y madres.

\begin{tabular}{|c|c|c|c|c|}
\hline & Sí & No & $\begin{array}{l}\text { Antes sí, pero } \\
\text { abandonó }\end{array}$ & $\left(x^{2}, p\right)$ \\
\hline \multicolumn{5}{|c|}{ Influencia del nivel educativo de los padres en la actividad practicada } \\
\hline Sin estudios & $5(1.1 \%)$ & $0(0.0 \%)$ & $5(2.4 \%)$ & \multirow{6}{*}{$10.8 ; p=0.545$} \\
\hline EPO & $116(26.4 \%)$ & $20(32.3 \%)$ & $55(26.8 \%)$ & \\
\hline FP grado medio & $57(13.0 \%)$ & $9(14.5 \%)$ & $27(13.2 \%)$ & \\
\hline ESO & $72(16.4 \%)$ & $13(21.0 \%)$ & $38(18.5 \%)$ & \\
\hline FP grado superior & $82(18.7 \%)$ & $11(17.7 \%)$ & $44(21.5 \%)$ & \\
\hline Estudios universitarios & $107(24.4 \%)$ & $9(14.5 \%)$ & $36(17.6 \%)$ & \\
\hline \multicolumn{5}{|c|}{ Influencia del nivel educativo de las madres en la actividad practicada } \\
\hline Sin estudios & $3(0.6 \%)$ & $1(1.3 \%)$ & $2(0.8 \%)$ & \multirow{6}{*}{$30.1 ; p=0.037$} \\
\hline EPO & $97(18.0 \%)$ & $18(23.4 \%)$ & $72(27.8 \%)$ & \\
\hline FP grado medio & $81(15.0 \%)$ & $11(14.3 \%)$ & $43(16.6 \%)$ & \\
\hline ESO & $93(17.3 \%)$ & $17(22.0 \%)$ & $58(22.4 \%)$ & \\
\hline FP grado superior & $118(21.8 \%)$ & $15(19.5 \%)$ & $35(13.5 \%)$ & \\
\hline Estudios universitarios & $147(27.3 \%)$ & $15(19.5 \%)$ & $49(18.9 \%)$ & \\
\hline
\end{tabular}

Fuente: elaboración propia; EPO: educación primaria obligatoria; ESO: educación secundaria obligatoria; FP: formación profesional.

En la Tabla 2, se pueden encontrar los valores de la estadística descriptiva y el análisis chi cuadrado para la actividad física realizada por los padres, madres y adolescentes. Los resultados muestran diferencias significativas en el nivel de 
actividad física de los adolescentes varones en función de la práctica realizada por sus padres. En este sentido, los varones que más actividad física realizaban tenían padres más activos. Respecto a las mujeres adolescentes, no se han encontrado diferencias significativas atendiendo a la práctica de los padres ni de las madres.

Tabla 2 - Nivel de práctica de los adolescentes en función de la práctica de padres y madres.

\begin{tabular}{|c|c|c|c|c|c|c|}
\hline & \multicolumn{2}{|c|}{ Activo } & \multicolumn{2}{|c|}{ Sedentario } & \multicolumn{2}{|c|}{$\left(x^{2}, p\right)$} \\
\hline & Alumnos & Alumnas & Alumnos & Alumnas & Alumnos & Alumnas \\
\hline \multicolumn{7}{|c|}{ Práctica de los adolescentes respecto a la de los padres } \\
\hline Sí & $183(77.9 \%)$ & $184(57.3 \%)$ & $31(53.4 \%)$ & $41(43.6 \%)$ & \multirow{3}{*}{$\begin{array}{c}17.9 \\
p=0.001\end{array}$} & \multirow{3}{*}{$\begin{array}{c}7.3 ; \\
p=0.123\end{array}$} \\
\hline No & $11(4.7 \%)$ & $33(10.3 \%)$ & $2(3.4 \%)$ & $16(17.0 \%)$ & & \\
\hline $\begin{array}{l}\text { Antes sí, pero } \\
\text { abandonó }\end{array}$ & $41(17.4 \%)$ & $104(32.4 \%)$ & $25(43.2 \%)$ & $37(39.4 \%)$ & & \\
\hline \multicolumn{7}{|c|}{ Práctica de los adolescentes respecto a la de las madres } \\
\hline Sí & $207(77.0 \%)$ & $220(56.4 \%)$ & $57(64.0 \%)$ & $54(42.9 \%)$ & \multirow{3}{*}{$\begin{array}{c}6.1 ; \\
p=0.189\end{array}$} & \multirow{3}{*}{$\begin{array}{c}8.3 ; \\
p=0.215\end{array}$} \\
\hline No & $11(4.0 \%)$ & $43(11.0 \%)$ & $6(6.7 \%)$ & $17(13.5 \%)$ & & \\
\hline $\begin{array}{l}\text { Antes sí, pero } \\
\text { abandonó }\end{array}$ & $51(19.0 \%)$ & 127 (32.6\%) & $26(29.3 \%)$ & $55(43.6 \%)$ & & \\
\hline
\end{tabular}

Fuente: elaboración propia.

En cuanto a la relación entre la duración diaria de la práctica de los adolescentes, los padres y las madres, cabe destacar que no se encontraron diferencias significativas en la duración diaria de práctica de los chicos y chicas adolescentes, en función de la duración diaria de los padres $(x 2=23.0 ; p=0.287)$, ni de las madres $(x 2=29.0$; $p=0.070)$.

La Tabla 3, presenta la estadística descriptiva para la frecuencia de práctica de los adolescentes, en función de la frecuencia de práctica de sus padres y madres, así como los resultados del test chi cuadrado. Cabe destacar que la frecuencia de práctica de las mujeres adolescentes mostró diferencias significativas en función de la frecuencia de práctica de las madres. En este sentido, un alto porcentaje de las adolescentes que practicaban 2-3 días semanales, tenían madres que practicaban con esta frecuencia. Sobre los varones adolescentes, no se encontraron diferencias significativas respecto a la frecuencia de los padres ni de las madres.

En la Tabla 4 se encuentra la estadística descriptiva y el análisis chi cuadrado para la práctica deportiva de los adolescentes en función de los estereotipos de género de las madres. Cabe destacar que se hallaron diferencias significativas en la práctica de los chicos y chicas adolescentes al considerar los estereotipos de género de las madres. En este sentido, los varones reportaron niveles superiores de práctica cuando la puntuación del estereotipo deporte y género de las madres era de 9, 10 u 11. Sobre las mujeres adolescentes, los niveles de práctica fueron significativamente superiores cuando la puntuación del estereotipo actividad física y deporte asociados al género de las madres era de 10, 11 o 12, y cuando la puntuación del estereotipo creencias sobre la actividad física, deporte y género de las madres era de 4, 5, 6, 7 u 8. Por su parte, los estereotipos de los padres no tuvieron una influencia significativa ni en la práctica de los varones ( $x 2$ $=12.4-42.2 ; p=0.109-0.995)$, ni de las mujeres $(x 2=29.6-53.7 ; p=0.370-0.963)$. 
Tabla 3 - Frecuencia de práctica de los chicos y chicas adolescentes dependiendo de la frecuencia de práctica de los padres y madres.

\begin{tabular}{|c|c|c|c|c|c|c|c|c|c|c|c|c|c|c|}
\hline & \multicolumn{2}{|c|}{$<1$ día } & \multicolumn{2}{|c|}{1 día } & \multicolumn{2}{|c|}{ 2-3 días } & \multicolumn{2}{|c|}{ 4-5 días } & \multicolumn{2}{|c|}{6 días } & \multicolumn{2}{|c|}{ Diaria } & \multicolumn{2}{|c|}{$\left(x^{2}, p\right)$} \\
\hline & Alumno & Alumna & Alumno & Alumna & Alumno & Alumna & Alumno & Alumna & Alumno & Alumna & Alumno & Alumna & Alumno & Alumna \\
\hline \multicolumn{15}{|c|}{ Frecuencia de práctica de los adolescentes respecto a la de los padres } \\
\hline 1 día & $0(0.0 \%)$ & $\begin{array}{c}15 \\
(18.8 \%)\end{array}$ & $\begin{array}{c}4 \\
(16.7 \%)\end{array}$ & $\begin{array}{c}9 \\
(21.4 \%)\end{array}$ & $\begin{array}{c}6 \\
(5.4 \%)\end{array}$ & $\begin{array}{c}16 \\
(11.0 \%)\end{array}$ & $\begin{array}{c}2 \\
(3.6 \%)\end{array}$ & $\begin{array}{c}7 \\
(10.1 \%)\end{array}$ & $\begin{array}{c}0 \\
(0.0 \%)\end{array}$ & $\begin{array}{c}1 \\
(5.9 \%)\end{array}$ & $\begin{array}{c}0 \\
(0.0 \%)\end{array}$ & $\begin{array}{c}1 \\
(7.1 \%)\end{array}$ & \multirow{4}{*}{$\begin{array}{c}43.9 ; \\
p=0.144\end{array}$} & \multirow{4}{*}{$\begin{array}{c}46.2 \\
p=0.097\end{array}$} \\
\hline $\begin{array}{l}4-5 \\
\text { días }\end{array}$ & $\begin{array}{c}14 \\
(25.5 \%)\end{array}$ & $\begin{array}{c}20 \\
(25.0 \%)\end{array}$ & $\begin{array}{c}7 \\
(29.2 \%)\end{array}$ & $\begin{array}{c}5 \\
(11.9 \%)\end{array}$ & $\begin{array}{c}44 \\
(39.6 \%)\end{array}$ & $\begin{array}{c}29 \\
(19.9 \%)\end{array}$ & $\begin{array}{c}32 \\
(58.2 \%)\end{array}$ & $\begin{array}{c}18 \\
(26.1 \%)\end{array}$ & $\begin{array}{c}6 \\
(40.0 \%)\end{array}$ & $\begin{array}{c}5 \\
(29.4 \%)\end{array}$ & $\begin{array}{c}8 \\
(66.7 \%)\end{array}$ & $\begin{array}{c}3 \\
(21.4 \%)\end{array}$ & & \\
\hline 6 días & $\begin{array}{c}0 \\
(0.0 \%)\end{array}$ & $\begin{array}{c}0 \\
(0.0 \%)\end{array}$ & $\begin{array}{c}0 \\
(0.0 \%)\end{array}$ & $\begin{array}{c}0 \\
(0.0 \%)\end{array}$ & $\begin{array}{c}1 \\
(0.9 \%)\end{array}$ & $\begin{array}{c}1 \\
(0.7 \%)\end{array}$ & $\begin{array}{c}1 \\
(1.8 \%)\end{array}$ & $\begin{array}{c}0 \\
(0.0 \%)\end{array}$ & $\begin{array}{c}0 \\
(0.0 \%)\end{array}$ & $\begin{array}{c}0 \\
(0.0 \%)\end{array}$ & $\begin{array}{c}0 \\
(0.0 \%)\end{array}$ & $\begin{array}{c}0 \\
(0.0 \%)\end{array}$ & & \\
\hline Diaria & $\begin{array}{c}1 \\
(1.8 \%)\end{array}$ & $\begin{array}{c}0 \\
(0.0 \%)\end{array}$ & $\begin{array}{c}0 \\
(0.0 \%)\end{array}$ & $\begin{array}{c}0 \\
(0.0 \%)\end{array}$ & $\begin{array}{c}3 \\
(2.7 \%)\end{array}$ & $\begin{array}{c}1 \\
(0.7 \%)\end{array}$ & $\begin{array}{c}0 \\
(0.0 \%)\end{array}$ & $\begin{array}{c}0 \\
(0.0 \%)\end{array}$ & $\begin{array}{c}1 \\
(6.7 \%)\end{array}$ & $\begin{array}{c}0 \\
(0.0 \%)\end{array}$ & $\begin{array}{c}1 \\
(8.3 \%)\end{array}$ & $\begin{array}{c}1 \\
(7.1 \%)\end{array}$ & & \\
\hline$<1$ día & $\begin{array}{c}2 \\
(2.4 \%)\end{array}$ & $\begin{array}{c}0 \\
(0.0 \%)\end{array}$ & $\begin{array}{c}1 \\
(7.1 \%)\end{array}$ & $\begin{array}{c}1 \\
(5.0 \%)\end{array}$ & $\begin{array}{c}5 \\
(3.4 \%)\end{array}$ & $\begin{array}{c}3 \\
(1.6 \%)\end{array}$ & $\begin{array}{c}1 \\
(1.5 \%)\end{array}$ & $\begin{array}{c}2 \\
(2.1 \%)\end{array}$ & $\begin{array}{c}0 \\
(0.0 \%)\end{array}$ & $\begin{array}{c}1 \\
(3.4 \%)\end{array}$ & $\begin{array}{c}0 \\
(0.0 \%)\end{array}$ & $\begin{array}{c}0 \\
(0.0 \%)\end{array}$ & \multirow{6}{*}{$\begin{array}{c}29.5 \\
p=0.729\end{array}$} & \multirow{6}{*}{$\begin{array}{c}53.6 \\
p=0.023\end{array}$} \\
\hline 1 día & $\begin{array}{c}4 \\
(4.8 \%)\end{array}$ & $\begin{array}{c}19 \\
(18.4 \%)\end{array}$ & $\begin{array}{c}2 \\
(14.3 \%)\end{array}$ & $\begin{array}{c}4 \\
(20.0 \%)\end{array}$ & $\begin{array}{c}5 \\
(3.4 \%)\end{array}$ & $\begin{array}{c}24 \\
(12.7 \%)\end{array}$ & $\begin{array}{c}4 \\
(6.0 \%)\end{array}$ & $\begin{array}{c}11 \\
(11.7 \%)\end{array}$ & $\begin{array}{c}1 \\
(7.7 \%)\end{array}$ & $\begin{array}{c}3 \\
(10.3 \%)\end{array}$ & $\begin{array}{c}0 \\
(0.0 \%)\end{array}$ & $\begin{array}{c}1 \\
(8.3 \%)\end{array}$ & & \\
\hline $\begin{array}{l}2-3 \\
\text { días }\end{array}$ & $\begin{array}{c}47 \\
(56.0 \%)\end{array}$ & $\begin{array}{c}64 \\
(62.1 \%)\end{array}$ & $\begin{array}{c}7 \\
(50.0 \%)\end{array}$ & $\begin{array}{c}12 \\
(60.0 \%)\end{array}$ & $\begin{array}{c}68 \\
(45.6 \%)\end{array}$ & $\begin{array}{c}124 \\
(65.6 \%)\end{array}$ & $\begin{array}{c}33 \\
(49.3 \%)\end{array}$ & $\begin{array}{c}53 \\
(56.4 \%)\end{array}$ & $\begin{array}{c}6 \\
(46.2 \%)\end{array}$ & $\begin{array}{c}20 \\
(69.0 \%)\end{array}$ & $\begin{array}{c}3 \\
(50.0 \%)\end{array}$ & $\begin{array}{c}9 \\
(75.0 \%)\end{array}$ & & \\
\hline $\begin{array}{l}4-5 \\
\text { días }\end{array}$ & $\begin{array}{c}27 \\
(32.1 \%)\end{array}$ & $\begin{array}{c}19 \\
(18.4 \%)\end{array}$ & $\begin{array}{c}4 \\
(28.6 \%)\end{array}$ & $\begin{array}{c}3 \\
(15.0 \%)\end{array}$ & $\begin{array}{c}68 \\
(45.6 \%)\end{array}$ & $\begin{array}{c}38 \\
(20.1 \%)\end{array}$ & $\begin{array}{c}27 \\
(40.3 \%)\end{array}$ & $\begin{array}{c}26 \\
(27.7 \%)\end{array}$ & $\begin{array}{c}4 \\
(30.8 \%)\end{array}$ & $\begin{array}{c}3 \\
(10.3 \%)\end{array}$ & $\begin{array}{c}3 \\
(50.0 \%)\end{array}$ & $\begin{array}{c}2 \\
(16.7 \%)\end{array}$ & & \\
\hline 6 días & $\begin{array}{c}1 \\
(1.2 \%)\end{array}$ & $\begin{array}{c}0 \\
(0.0 \%)\end{array}$ & $\begin{array}{c}0 \\
(0.0 \%)\end{array}$ & $\begin{array}{c}0 \\
(0.0 \%)\end{array}$ & $\begin{array}{c}1 \\
(0.7 \%)\end{array}$ & $\begin{array}{c}0 \\
(0.0 \%)\end{array}$ & $\begin{array}{c}0 \\
(0.0 \%)\end{array}$ & $\begin{array}{c}1 \\
(1.1 \%)\end{array}$ & $\begin{array}{c}1 \\
(7.7 \%)\end{array}$ & $\begin{array}{c}2 \\
(6.9 \%)\end{array}$ & $\begin{array}{c}0 \\
(0.0 \%)\end{array}$ & $\begin{array}{c}0 \\
(0.0 \%)\end{array}$ & & \\
\hline Diaria & $\begin{array}{c}3 \\
(3.6 \%)\end{array}$ & $\begin{array}{c}1 \\
(1.0 \%)\end{array}$ & $\begin{array}{c}0 \\
(0.0 \%)\end{array}$ & $\begin{array}{c}0 \\
(0.0 \%)\end{array}$ & $\begin{array}{c}2 \\
(1.3 \%)\end{array}$ & $\begin{array}{c}0 \\
(0.0 \%)\end{array}$ & $\begin{array}{c}2 \\
(3.0 \%)\end{array}$ & $\begin{array}{c}1 \\
(1.1 \%)\end{array}$ & $\begin{array}{c}1 \\
(7.7 \%)\end{array}$ & $\begin{array}{c}0 \\
(0.0 \%)\end{array}$ & $\begin{array}{c}0 \\
(0.0 \%)\end{array}$ & $\begin{array}{c}0 \\
(0.0 \%)\end{array}$ & & \\
\hline
\end{tabular}

Fuente: elaboración propia 
Tabla 4 - Práctica deportiva de los adolescentes respecto a los estereotipos de género de las madres.

\begin{tabular}{|c|c|c|c|c|c|c|c|c|c|c|c|c|c|c|c|c|c|c|c|c|c|c|c|c|}
\hline \multicolumn{25}{|c|}{ Diferencias Asociadas al Género y su relación con la actividad física y el deporte (Puntuación) } \\
\hline & & 7 & 8 & 9 & 10 & 11 & 12 & 13 & 14 & 15 & 16 & 17 & 18 & 19 & 20 & 21 & 22 & 23 & 24 & 25 & 26 & 27 & 28 & $\left(x^{2}, p\right)$ \\
\hline \multirow{2}{*}{ Varones } & Sí practica & 15 & 20 & 30 & 41 & 23 & 26 & 23 & 17 & 18 & 18 & 8 & 2 & 5 & 3 & 2 & 0 & 1 & 1 & 0 & 0 & 0 & 0 & \multirow{2}{*}{29,$9 ; p=0,754$} \\
\hline & No practica & 11 & 6 & 13 & 7 & 13 & 9 & 10 & 6 & 9 & 1 & 1 & 1 & 2 & 0 & 1 & 0 & 0 & 0 & 0 & 0 & 0 & 0 & \\
\hline \multirow{2}{*}{ Mujeres } & Sí practica & 19 & 24 & 30 & 32 & 32 & 28 & 23 & 24 & 23 & 11 & 8 & 5 & 2 & 2 & 0 & 0 & 0 & 0 & 2 & 0 & 0 & 0 & \multirow{2}{*}{44,$6 ; p=0,814$} \\
\hline & No practica & 12 & 16 & 37 & 32 & 26 & 27 & 14 & 22 & 19 & 10 & 5 & 6 & 3 & 2 & 1 & 1 & 0 & 0 & 1 & 0 & 0 & 0 & \\
\hline \multicolumn{25}{|c|}{ Estereotipos sobre la actividad física y deporte asociados al género (Puntuación) } \\
\hline & & 5 & 6 & 7 & 8 & 9 & 10 & 11 & 12 & 13 & 14 & 15 & 16 & 17 & 18 & 19 & 20 & \multicolumn{3}{|c|}{$(x 2 . p)$} & & & & \\
\hline \multirow{2}{*}{ Varones } & Sí practica & 11 & 10 & 20 & 22 & 29 & 33 & 39 & 30 & 28 & 11 & 11 & 2 & 6 & 2 & 0 & 0 & \multirow{2}{*}{\multicolumn{3}{|c|}{$39.7 ; p=0.393$}} & & & & \\
\hline & No practica & 6 & 5 & 10 & 12 & 5 & 12 & 13 & 10 & 4 & 6 & 5 & 1 & 0 & 0 & 1 & 0 & & & & & & & \\
\hline \multirow{2}{*}{ Mujeres } & Sí practica & 12 & 14 & 17 & 27 & 23 & 46 & 36 & 34 & 17 & 15 & 16 & 3 & 4 & 1 & 0 & 0 & \multicolumn{3}{|c|}{$100.6 ; p<0.001$} & & & & \\
\hline & No practica & 11 & 14 & 18 & 28 & 30 & 26 & 26 & 36 & 15 & 16 & 9 & 2 & 1 & 1 & 1 & 0 & & & & & & & \\
\hline \multicolumn{25}{|c|}{ Deporte y Género (Puntuación) } \\
\hline & & 4 & 5 & 6 & 7 & 8 & 9 & 10 & 11 & 12 & 13 & 14 & 15 & 16 & \multicolumn{3}{|c|}{$\left(x^{2} \cdot p\right)$} & & & & & & & \\
\hline \multirow{2}{*}{ Varones } & Sí practica & 8 & 12 & 15 & 17 & 26 & 35 & 54 & 41 & 23 & 17 & 7 & 5 & 2 & \multirow{2}{*}{\multicolumn{3}{|c|}{$47.0 ; p=0.014$}} & & & & & & & \\
\hline & No practica & 3 & 3 & 5 & 6 & 14 & 7 & 8 & 15 & 11 & 11 & 8 & 1 & 2 & & & & & & & & & & \\
\hline Mujeres & No practica & 15 & 9 & 16 & 17 & 30 & 29 & 29 & 27 & 28 & 18 & 10 & 6 & 4 & & & & & & & & & & \\
\hline \multicolumn{25}{|c|}{ Las clases de Educación Física y el género (Puntuación) } \\
\hline & & 4 & 5 & 6 & 7 & 8 & 9 & 10 & 11 & 12 & 13 & 14 & 15 & 16 & \multicolumn{3}{|c|}{$\left(x^{2} \cdot p\right)$} & & & & & & & \\
\hline \multirow{2}{*}{ Varones } & Sí practica & 63 & 19 & 20 & 25 & 58 & 24 & 24 & 8 & 7 & 2 & 2 & 0 & 1 & \multirow{2}{*}{\multicolumn{3}{|c|}{$12.9 ; p=0.967$}} & & & & & & & \\
\hline & No practica & 30 & 4 & 9 & 8 & 22 & 7 & 5 & 3 & 1 & 0 & 1 & 0 & 0 & & & & & & & & & & \\
\hline \multirow{2}{*}{ Mujeres } & Sí practica & 56 & 28 & 18 & 27 & 60 & 23 & 24 & 11 & 11 & 2 & 2 & 1 & 1 & & $2 ; p=c$ & .912 & & & & & & & \\
\hline & No practica & 53 & 19 & 24 & 13 & 65 & 21 & 16 & 9 & 6 & 2 & 1 & 0 & 2 & & & & & & & & & & \\
\hline & & Sreen & cias & sobre & la a & ivida & d fís & $\mathrm{ca}, \mathrm{de}$ & eporte & ey gé & Enero & (Pun & tuaci & ón) & & & & & & & & & & \\
\hline & & 4 & 5 & 6 & 7 & 8 & 9 & 10 & 11 & 12 & 13 & 14 & 15 & 16 & & $\left(x^{2} \cdot \mathrm{p}\right.$ & & & & & & & & \\
\hline Varones & Sí practica & 40 & 30 & 40 & 41 & 38 & 29 & 21 & 7 & 6 & 3 & 0 & 0 & 1 & & & & & & & & & & \\
\hline varities & No practica & 18 & 14 & 22 & 14 & 11 & 4 & 5 & 1 & 1 & 1 & 0 & 0 & 0 & & $.5 ; p=c$ & .966 & & & & & & & \\
\hline Muriore & Sí practica & 52 & 35 & 46 & 35 & 46 & 26 & 22 & 3 & 3 & 0 & 1 & 0 & 0 & & $.2 ; p<$ & 0.001 & & & & & & & \\
\hline iviujeres & No practica & 43 & 38 & 39 & 30 & 41 & 17 & 15 & 7 & 5 & 1 & 0 & 0 & 0 & & & & & & & & & & \\
\hline
\end{tabular}

Fuente: elaboración propia

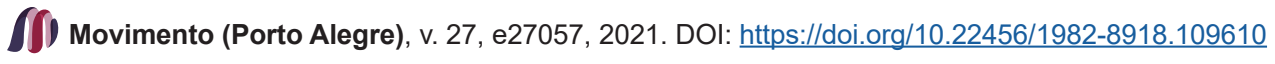


En la Tabla 5, se presenta el análisis correlacional de los estereotipos de género de los adolescentes, padres y madres. La mayoría de las dimensiones no mostraron una correlación significativa, siendo además las correlaciones encontradas muy débiles.

Tabla 5 - Análisis correlacional de los estereotipos de género de adolescentes, padres y madres.

\begin{tabular}{ccc}
\hline & & $\begin{array}{c}\text { Estereotipos de género de los } \\
\text { adolescentes }\end{array}$ \\
\hline $\begin{array}{c}\text { Diferencias asociadas al género y su relación } \\
\text { con la actividad física y el deporte }\end{array}$ & $\begin{array}{c}\text { Hombre } \\
\text { Mujer }\end{array}$ & $r=0.045 ; p=0.162$ \\
Deporte y género & Hombre & $r=0.055 ; p=0.085$ \\
\hline \multirow{2}{*}{ Actividad física y deporte asociados al género } & Mujer & $r=0.535$ \\
Creencias sobre la actividad física. & Mombre & $r=0.066 ; p=0.040$ \\
\hline deporte y género & Hombre & $r=0.072 ; p=0.025$ \\
\hline \multirow{2}{*}{ Las clases de educación física y el género } & Mujer & $r=0.071 ; p=0.027$ \\
& Mombre & $r=0.110 ; p=0.001$ \\
\hline
\end{tabular}

Fuente: elaboración propia

\section{DISCUSIÓN}

El objetivo principal del estudio fue analizar la influencia de los estereotipos de género y el nivel de práctica deportiva de los progenitores sobre la práctica deportiva de sus hijos. En cuanto a los estereotipos de los progenitores, cabe destacar la influencia de los estereotipos de género de las madres sobre la práctica de sus hijos, independientemente del sexo de los mismos. Las investigaciones previas muestran que los estereotipos de género de los progenitores pueden influir sobre la participación deportiva de sus hijos, así como en sus estereotipos de género (BOICHÉ et al., 2014), si bien en estos estudios no se ha analizado la influencia en función del sexo de los progenitores. En base a los resultados de la presente investigación, son las madres el factor más influyente en esta relación. Cabe destacar que las chicas parecen estar más influidas que los chicos por los estereotipos de sus progenitores, lo que corrobora las investigaciones previas realizadas por Henriksen et al. (2016). En base a esto, sería conveniente realizar programas de intervención con las madres para abordar este tópico, especialmente cuando tienen hijas.

Un hallazgo importante de la presente investigación fue la falta de influencia de los estereotipos de los progenitores sobre los estereotipos de los adolescentes. Es relevante porque la opinión de los padres y madres en cuanto al nivel y tipo de práctica deportiva en función del sexo no estaría presente en los adolescentes, lo que indicaría un avance en las nuevas generaciones para alcanzar la igualdad en el deporte. Estos resultados son contrarios a los hallados por Pellett e Ignico (1993) en una muestra de estudiantes de educación primaria en la que establecieron relación significativa entre los estereotipos de los progenitores y los hijos. Esto podría deberse a que la adolescencia es la etapa en la que las formas de actuar y las opiniones 
de los progenitores podrían ir perdiendo relevancia en favor de las opiniones de sus amigos (GATOUILLAT; GRIFFET; TRAVERT, 2019) o porque las propuestas de concienciación sobre la igualdad en el deporte que se han desarrollado en los últimos años están provocando modificaciones en la percepción de estereotipos de género en el deporte (BALISH et al., 2016; NORMAN, 2015). Tras estos prometedores resultados, investigaciones futuras podrían abordar la influencia que tienen los otros agentes sociales como los iguales en la creación de unos determinados estereotipos de género durante la adolescencia, y analizar si se producen modificaciones respecto a los estereotipos a edades más tempranas, lo que confirmaría que la adolescencia es un periodo clave para la asimilación de los estereotipos de género (BOICHÉ et al., 2014).

A pesar de que su influencia sobre los estereotipos de género parece ser reducida, los progenitores siguen siendo fundamentales por la influencia que ejercen en los adolescentes para comenzar a practicar actividades deportivas. Estos resultados siguen la misma línea de las investigaciones previas (BRINGOLF-ISLER et al., 2018; CHENG; MENDONÇA; FARIAS-JUNIOR, 2014; HENRIKSEN et al., 2016; LISINSKIENE; JUSKELIENE, 2019; MENDONÇA et al., 2014). Por tanto, se plantea la hipótesis de que realizar intervenciones tempranas con los progenitores podría ser beneficioso para concienciarlos sobre el rol que desempeñan en la práctica deportiva de sus hijos, lo que podría favorecer que la influencia posterior ejercida sobre los adolescentes fuese beneficiosa; teniendo que ser esta hipótesis contrastada en futuras investigaciones.

Respecto a la importancia del nivel educativo de los progenitores, los resultados indican que los adolescentes tenían niveles superiores de actividad física cuando sus madres tenían un nivel educativo más elevado. Estos resultados difieren de los hallados en investigaciones previas, en las que los adolescentes que más actividades físicas practicaban tenían padres y madres con niveles educativos superiores (MAGALHAES; PEREIRA DE PINA; PEREIRA RAMOS, 2017; STEENHOLT et al., 2018), o solo padres (BRINGOLF-ISLER et al., 2018), o no encontraron influencia del nivel educativo de los progenitores sobre la práctica de los adolescentes (SANZARAZURI et al., 2018). Las diferencias en los hallazgos de la presente investigación con los estudios previos podrían deberse a que la tasa de empleo es superior en los hombres respecto a las mujeres (INSTITUTO NACIONAL DE ESTADÍSTICA, 2020), lo que conllevaría un mayor tiempo en el hogar de las mismas y una mayor influencia en la vida de sus hijos, incluida la práctica deportiva.

En cambio, el nivel de práctica deportiva de los padres, pero no el de las madres, es determinante en la práctica deportiva de los hijos varones. En este sentido, los chicos adolescentes que reportaban niveles de práctica deportiva superiores tenían padres más activos, lo que corrobora los estudios previos en los que se encontró que la práctica deportiva de los progenitores influye sobre la práctica de los adolescentes del mismo sexo (BRINGOLF-ISLER et al., 2018; CHENG; MENDONÇA; FARIAS-JUNIOR., 2014). Sin embargo, ni padres ni madres parecen ser influyentes en la frecuencia y duración de práctica de los varones, contradiciendo lo encontrado en estudios previos en los que se encontraba una influencia de los 
progenitores también en este ámbito (CHENG; MENDONÇA; FARIAS-JUNIOR, 2014; SÁNCHEZ-ZAMORANO et al., 2019). Esto podría explicarse al considerar que la frecuencia y duración de la práctica de los adolescentes suele ser superior a la de los progenitores, por lo que la influencia de estos es mínima en este sentido. La clave por tanto estaría en que los progenitores practiquen y otorguen importancia a las actividades físicas, porque de no hacerlo, es más probable que los adolescentes no se adhieran a la práctica deportiva (SANZ-ARAZURI et al., 2018).

Respecto a la práctica de las chicas adolescentes, no se encontró una relación de la misma con la práctica de ninguno de sus progenitores. Los resultados difieren de los encontrados en una investigación previa que encontró una influencia de la práctica de las madres sobre la práctica de las chicas en la adolescencia (BRINGOLFISLER et al., 2018). Esto podría deberse a que las adolescentes no han encontrado una actividad que les guste, siendo este uno de los principales motivos que las lleva a no practicar (CRANE; TEMPLE, 2014), o a que no encuentran iguales con las que practicar y hacerlo con las madres podría no reportarles el mismo grado de satisfacción por lo que podrían preferir dedicar su tiempo a actividades no deportivas en compañía de sus iguales (DIAS; LOCH; VAZ-RONQUE, 2015). No obstante, la frecuencia de práctica de las madres se relacionó significativamente con una mayor frecuencia de práctica de las hijas. Estos resultados siguen la línea de las investigaciones previas, ya que en estas se indicó que había modificaciones en la frecuencia y duración de las chicas al considerar la de las madres (CHENG; MENDONÇA; FARIAS-JUNIOR, 2014; SÁNCHEZ-ZAMORANO et al., 2019). Se puede extraer de estos resultados que una vez que las chicas practican, las madres son un referente para establecer el volumen de la misma, lo que podría tener una gran relevancia para la salud de las adolescentes (RODRIGUES; PADEZ; MACHADO-RODRIGUES, 2018).

Respecto a las limitaciones del presente estudio, cabe destacar que, aunque se informó a los participantes del modo correcto de cumplimentación del material, los cuestionarios fueron cumplimentados por los adolescentes y progenitores en sus domicilios y sin supervisión del equipo investigador durante su realización. Aunque el tamaño de la muestra es considerable, son necesarias investigaciones futuras en las que se utilicen los mismos cuestionarios y permitan contrastar los resultados obtenidos con mayor vigilancia epistémica.

\section{CONCLUSIÓN}

En conclusión, el presente estudio demuestra que los estereotipos de género de las madres influyen en los niveles de práctica deportiva de los adolescentes, pero no en sus estereotipos de género; el nivel educativo de las madres determina la práctica de actividad física de sus hijos, independientemente del sexo; mientras que los padres son más influyentes que las madres en que los adolescentes varones practiquen, si bien las madres parecen influir más en la frecuencia de práctica de las chicas. 


\section{REFERENCIAS}

ALVARIÑAS-VILLAVERDE, Myriam; FERNÁNDEZ-VILLARINO, Maria de los Ángeles; LÓPEZ-VILLAR, Cristina. Actividad física y percepciones sobre deporte y género. Revista de Investigación en Educación, v. 6, p. 113-122, ene. 2009.

ALVARIÑAS-VILLAVERDE, Myriam et al. Masculine, Feminine and neutral sports:

Extracurricular sport modalities in practice. Journal of Human Sport and Exercise, v. 12, n. 4, p. 1278-1288, 2017.

BALISH, Shea et al. Gender equality predicts leisure-time physical activity: Benefits for both sexes across 34 countries. Cogent Psychology, v. 3, n. 1, p. 1-7, Mar./Mayo, 2016.

BOICHÉ, Julie et al. Social antecedents and consequences of gender-sport stereotypes during adolescence. Psychology of Women Quarterly, v. 38, n. 2, p. 259-274, Jun. 2014.

BRINGOLF-ISLER, Bettina et al. Objectively measured physical activity in populationrepresentative parent-child pairs: parental modeling matters and is context-specific. BMC Public Health, v. 18, n. 1, p. 1-15, Ago. 2018.

CHALABAEV, Aïna et al. The influence of sex stereotypes and gender roles on participation and performance in sport and exercise: Review and future directions. Psychology of Sport and Exercise, v. 14, n. 2, p, 136-144, Mar. 2013.

CHENG, Luanna; MENDONCA, Gerfeson; FARIAS-JUNIOR, José Cazuza. Physical activity in adolescents: analysis of the social influence of parents and friends. Jornal de Pediatria, v. 90, n. 1 , p. $35-41,2014$.

CRANE, Jeff; TEMPLE, Viviene. A systematic review of dropout from organized sport among children and youth. European Physical Education Review, v. 21, n. 1, p. 114-131, Oct. 2014.

DIAS, Douglas; LOCH, Mathias; VAZ-RONQUE, Enio. Perceived barriers to leisure-time physical activity and associated factors in adolescents. Ciência \& Saúde Coletiva, v. 20, n. 11, p. 3339-3350, nov. 2015.

FERNÁNDEZ-PRIETO, Isabel; GINÉ-GARRIGA, María; VÉLEZ, Olga. Barreras y motivaciones percibidas por adolescentes en relación con la actividad física. Estudio cualitativo a través de grupos de discusión. Revista Española de Salud Pública, v. 93, p. 1-12, ago. 2019.

GATOUILLAT, Colin; GRIFFET, J; TRAVERT, Maxime. Navigating the circles of social life: understanding pathways to sport drop-out among French teenagers. Sport, Education and Society, v. 25, n. 1, p. 1-13, July.2019.

GONZALO-ALMOROX, Eduardo; URBANOS-GARRIDO, Rosa. Decomposing socioeconomic inequalities in leisure-time physical inactivity: the case of Spanish children. International Journal for Equity in Health, v. 15, p. 1-10, Jul. 2016.

GUAGLIANO, Justin et al. Do coaches perceive themselves as influential on physical activity for girls in organized youth sport? PLOS ONE, v. 9, n. 9, p. 1-14, Sept. 2014.

HENRIKSEN, Pia et al. Physical activity among adolescents: the role of various kinds of parental support. Scandinavian Journal of Medicine \& Science in Sports, v. 26, n. 8, p. 1-6, Aug. 2016.

INSTITUTO NACIONAL DE ESTADÍSTICA. Tasas de actividad, paro y empleo por provincia y sexo. Madrid: Instituto Nacional de Estadística, 2020. 
LISINSKIENE, Ausra; JUSKELIENE, Vida. Links between adolescents' engagement in physical activity and their attachment to mothers, fathers, and peers. International Journal of Environmental Research and Public Health, v. 16, n. 5, p. 1-11, Mar. 2019.

LÓPEZ-ALBALÁ, Elena. Mujeres deportistas españolas: estereotipos de género en los medios de comunicación. Sociologiados. Revista de Investigación Social, v. 1, n. 2, p. 87110, 2016.

MAGALHAES, Alexandre Pedro; PEREIRA DE PINA, Maria de Fátima; PEREIRA RAMOS, Elisabete. The role of urban environment, social and health determinants in the tracking of leisure-time physical activity throughout adolescence. Journal of Adolescent Health, v. 60, n. 1, 100-106, Jan. 2017.

MARTÍNEZ-ALMECIJA, Alfredo; MUÑOZ-GARCÍA, Joaquín; PASCUAL-ACOSTA, Antonio. Tamaño de muestra y precisión estadística. Almería: Universidad de Almería, 2004.

MENDONÇA, Gerfeson et al. Physical activity and social support in adolescents: a systematic review. Health Education Research, v. 29, n. 5, p. 822-839, Oct. 2014.

NORMAN, Leanne. The impact of an "equal opportunities" ideological framework on coaches' knowledge and practice. International Review for the Sociology of Sport, v. 51, n. 8, p. 975-1004, Jan. 2015.

\section{ORGANIZACIÓN MUNDIAL DE LA SALUD. Recomendaciones mundiales sobre actividad física para la salud. Ginebra, 2010.}

PELLETT, Tracy; IGNICO, Arlene. Relationship between children's and parents stereotyping of physical activities. Perceptual and Motor Skills, v. 77, p. 1283-1289, Dec. 1993.

RENNINGER, Marius et al. Associations between accelerometry measured physical activity and sedentary time and the metabolic syndrome: a meta-analysis of more than 6000 children and adolescents. Pediatric Obesity, v. 15, n. 1, p. 1-9, Jan. 2020.

RODRIGUES, Daniela; PADEZ, Cristina; MACHADO-RODRIGUES, Aristides. Active parents, active children: The importance of parental organized physical activity in children's extracurricular sport participation. Journal of Child Health Care, v. 22, n. 1, p. 159-170, Mar. 2018

RODRIGUES, Daniela; PADEZ, Cristina; MACHADO-RODRIGUES, Aristides. Parental perception of barriers to children's participation in sports: biological, social, and geographic correlates of Portuguese children. Journal of Physical Activity and Health, v. 16, n. 8, p. 595-600, Aug. 2019.

SÁNCHEZ-ZAMORANO, Luisa María et al. Perception of parents' physical activity as a positive model on physical activity of adolescents. Preventive Medicine, v. 127, p. 1-7, Aug. 2019.

SANZ-ARAZURI, Eva et al. Parental influence on adolescent adherence to physical-sport practice. RETOS. Nuevas Tendencias en Educación Física, Deporte y Recreación, v. 33, p. 185-189, 2018.

STEENHOLT, Carina et al. School and class-level variations and patterns of physical activity: a multilevel analysis of Danish high school students. BMC Public Health, v. 18, n. 1, p. 1-11, Feb. 2018

TAYLOR, lan et al. Motivational predictors of physical education students' effort, exercise intentions, and leisure-time physical activity: A multilevel linear growth analysis. Journal of Sport and Exercise Psychology, v. 32, n. 1, p. 99-120, Feb. 2010. 
TOFFOLETTI, Kim; THORPE, Holly. Female athletes' self-representation on social media: A feminist analysis of neoliberal marketing strategies in "economies of visibility". Feminism and Psychology, v. 28, n. 1, p. 11-31, Feb. 2018.

VANDENBROUCKE, Jan et al. Strengthening the Reporting of Observational Studies in Epidemiology (STROBE): explanation and elaboration. International Journal of Surgery, $\mathrm{v}$. 12, n. 12, p. 1500-1524, Dec. 2014.

VERA, Juan; ARREBOLA, Inmaculada; GARCÍA, Nahum. Gender and its relationship with the practice of physical activity and sport. Apunts. Educación Física y Deportes, v. 132, p. 123-141, 2018

WILK, Piotr et al. Exploring the effect of parental influence on chidlren's physical activity: The mediating role of children's perceptions of parental support. Preventive Medicine, v. 106, p. 79-85, 2018 
Resumo: A importância dos pais na atividade física dos seus filhos durante a adolescência foi previamente afirmada, mas poucos estudos determinaram quais são as variáveis mais determinantes. O objetivo do presente estudo foi analisar a influência dos estereótipos de gênero, o nível educacional e o nível de prática desportiva dos pais sobre a prática desportiva dos seus filhos. A amostra foi composta por 965 adolescentes e 1599 pais da Região de Múrcia. Os resultados mostraram diferenças na prática desportiva dos adolescentes ao considerar o nível educacional das mães, a atividade física realizada pelos pais para os homens, a frequência da prática das mães para as mulheres e os estereótipos de gênero das mães. Como conclusões, é de notar que os estereótipos de gênero e o nível de educação dos pais influenciaram a prática do desporto pelos adolescentes.

Palavras chave: Exercício físico. Adolescente. Estereotipagem de gênero. Pais.

Abstract: The importance of parents in the physical activity of their children during adolescence has been previously established, but few studies have determined which are the most important variables. This study analyzes the influence of gender stereotypes, educational level, and the level of parents' sports practice on their children's practice. The sample included 965 adolescents and 1,599 parents from the Region of Murcia. The results showed differences in the sports practice of the adolescents when considering the educational level of their mothers, the physical activity carried out by the fathers on male children, the frequency of practice of the mothers for female children and gender stereotypes of the mothers. It found that gender stereotypes and parents' educational level influenced adolescents' sports practice.

Keywords: Exercise. Adolescent. Gender stereotyping. Parents. 


\section{LICENCIA DE USO}

Este es un artículo publicado em Open Access bajo la licencia Creative Commons Attribution 4.0 International (CC BY 4.0), que permite su uso, distribución y reproducción en cualquier medio, siempre que se cite correctamente la obra original. Más información en: http://creativecommons.org/licenses/by/4.0

\section{CONFLICTO DE INTERESES}

Los autores declaran que no existe ningún conflicto de intereses en este trabajo.

\section{CONTRIBUCIONES DE LOS AUTORES}

Adrián Mateo: Conservación de datos, análisis formal, investigación, redacción del borrador original.

Raquel Vaquero: Conceptualización, análisis formal, investigación, redacción del borrador original.

Lucía Abenza: Conceptualización, análisis formal, investigación, redacción del borrador original.

Sonia María Martínez: Conceptualización, metodología, revisión de la redacción y edición.

Ana María Gallardo: Conceptualización, metodología, revisión de la redacción y edición.

Alejandro Leiva: Conceptualización, metodología, revisión de la redacción y edición.

Antonio Sánchez Pato: Conceptualización, obtención de fondos, metodología, administración del Proyecto, supervisión, revisión y edición de la redacción.

\section{FINANCIACIÓN}

Este trabajo se llevó a cabo con el apoyo de la Unión Europea con la denominación de proyecto A.G.E.S: Addressing Gender Equity in Sport (603379-EPP-1-2018-1-ITSPO-SSCP).

\section{ÉTICA DE LA INVESTIGACIÓN}

El trabajo ha sido apreciado y aprobado en Comité de Ética de Investigación (CEP) de Universidad Católica San Antonio de Murcia, Protocolo Código CE071924.

\section{CÓMO CITAR}

MATEO-ORCAJADA, Adrián; VAQUERO-CRISTÓBAL, Raquel; ABENZA-CANO, Lucía; MARTíNEZ-CASTRO, Sonia María; GALLARDO-GUERRERO, Ana María; LEIVA-ARCAS, Alejandro; SÁNCHEZ-PATO, Antonio. Influencia del género, nivel educativo y práctica deportiva parentales sobre hábitos deportivos en escolares. Movimento (Porto Alegre), v. 27, e27057, ene./dic. 2021. Disponible en: https:// seer.ufrgs.br/Movimento/article/view/109610. Consultado en: [día] [mes abreviado]. [año]. DOI: https://doi.org/10.22456/1982-8918.109610 


\section{RESPONSABILIDAD EDITORIAL}

Alex Branco Fraga*, Elisandro Schultz Wittizorecki*, Ileana Wenetz ${ }^{* *}$, Ivone Job*, Mauro Myskiw*, Raquel da Silveira*

*Universidad Federal de Rio Grande do Sul, Escuela de Educación Física, Fisioterapia y Danza, Porto Alegre, RS, Brasil.

*Universidad Federal de Espirito Santo, Vitoria, ES, Brasil. 